\title{
INOVAÇÃO, CROWDSOURCING E CROWD- DESIGN: POSSIBILIDADES DE ATUAÇÃO NO CENÁRIO CONTEMPORÂNEO
}

INNOVATION, CROWDSOURCING AND CROWD-DESIGN: POSSIBILITIES OF ACTION IN THE CONTEMPORARY SCENE
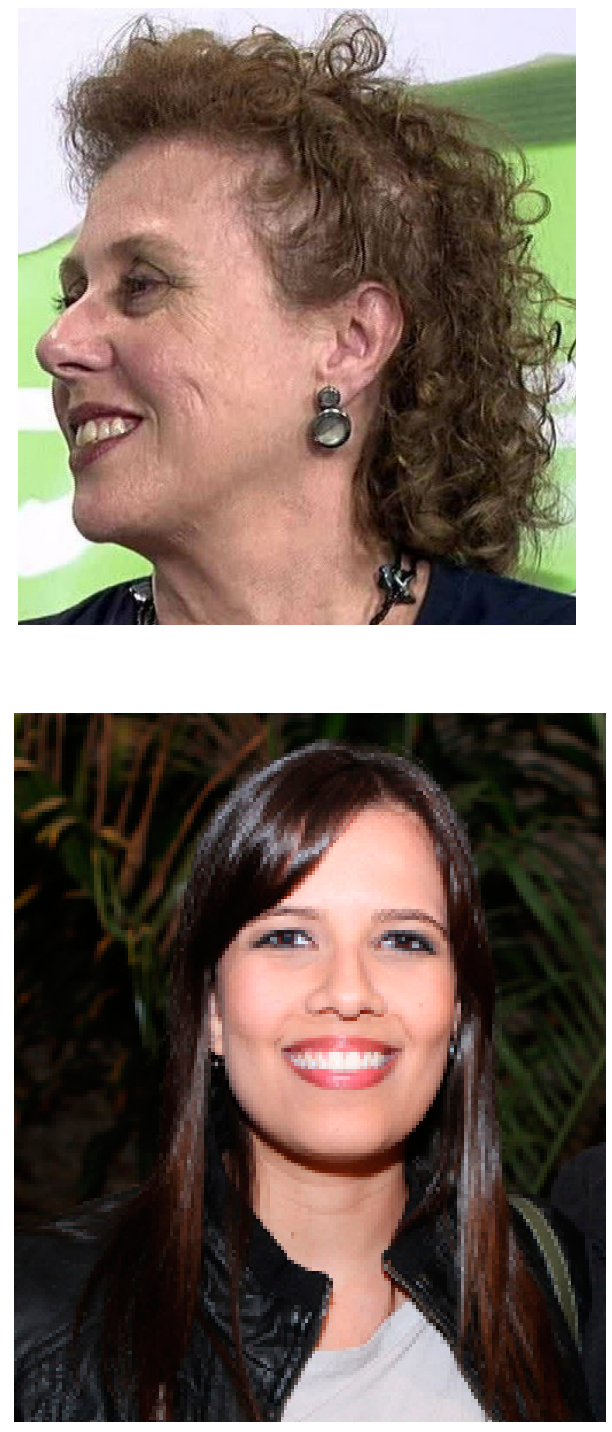

\section{Paula da Cruz Landim}

Pós-doutorado em Design pela Universidade de Arte e Design de Helsinque (UIAH) Livre-Docente em Design de Produto pela Universidade Estadual Paulista Júlio de Mesquita Filho (UNESP) paula@faac.unesp.br

\section{Lílian Lago}

Mestranda em Design pela Universidade Estadual Paulista Júlio de Mesquita Filho (UNESP)

Programa de Pós-Graduação em Design (UNESP) lago.lilian@gmail.com 


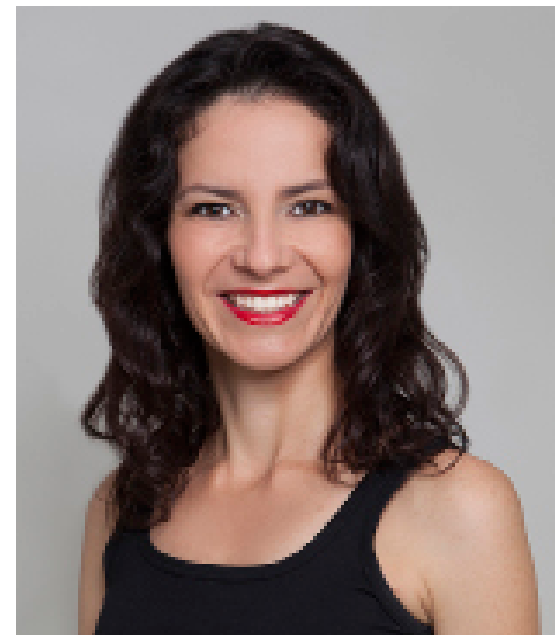

\section{Juliana Cristina de Angelo}

Mestranda em Design pela Universidade Estadual Paulista Júlio de Mesquita Filho (UNESP)

Programa de Pós-Graduação em Design (UNESP)

jul.angel@hotmail.com

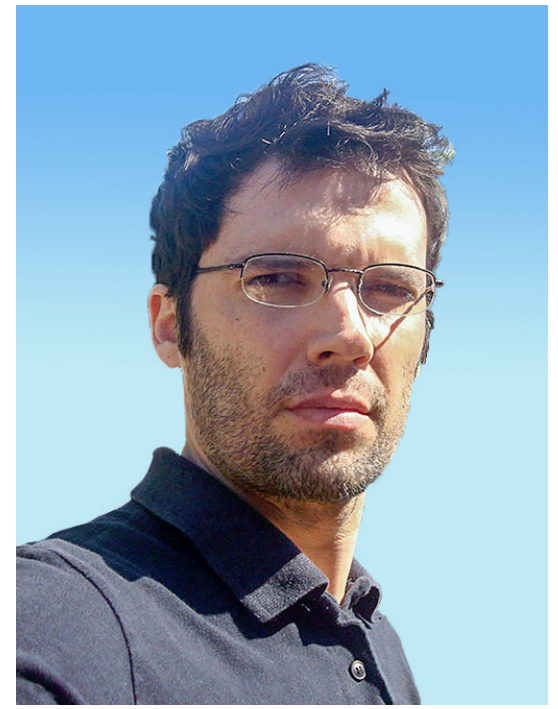

\section{Fabrício Mira}

Mestrando em Design pela Universidade Estadual Paulista Júlio de Mesquita Filho (UNESP) ProgramadePós-GraduaçãoemDesign(UNESP) fabriciomira@gmail.com

\section{RESUMO}

O século XXI é por muitos considerado a era das redes colaborativas. A indústria já não é mais o maior setor da economia e as pessoas já demonstram iniciativa em produzir o que é de interesse próprio ou comunitário. Mediante revisão de literatura sobre inovação, contemporaneidade, crowdsourcing e crowd-design e a apresentação de um case de design premiado, o Eatwell, um conjunto de mesa para pessoas com deficiências cognitivas, como doença de Alzhiemer, é possível compreender a importância da articulação de stakeholders para se fazer inovação hoje, sem depender de um grande investidor ou do interesse e liderança da indústria.

\section{PALAVRAS-CHAVE}

Inovação. Crowdsourcing. Crowd-design. 


\section{ABSTRACT}

The twenty-first century is widely considered the era of collaborative networks. Industry is no longer the largest sector of the economy and people have shown initiative in producing proper interest or community interest. From a literature review on innovation, contemporaneity, crowdsourcing and crowd-design and presentation of a case of award-winning design, Eatwell, tableware set for people with special need/Alzhiemer's, one can understand the importance of coordination of stakeholders to make innovation today without relying on a major investor or interest and leadership of industry.

\section{KEYWORDS}

Innovation. Crowdsourcing. Crowd-design.

\section{INTRODUÇÃO}

O cenário contemporâneo apresenta novos fatores de influência para a inovação. Atores sociais, tais como instituições, empresas, organizações nãolucrativas, cidadãos e suas associações, têm em mãos novas ferramentas e métodos para produzir inovação. Com o suporte das redes, o crowdsourcing e suas ações derivadas ganham espaço e alcançam ao design, dando origem ao chamado "crowd-design".

Para exemplificar a importância do relacionamento e do trabalho conjunto entre stakeholders foi escolhido o case de design Eatwell, um conjunto de mesa para pessoas com deficiências cognitivas, como doença de Alzheimer e demência, que ganhou o $1^{\circ}$ lugar no Design Challenge "Maximizing Independence for those with cognitive impairment" promovido pelo Stanford Center of Longevity. Tal projeto é um exemplo de design centrado no usuário e um produto inovador por seu desenho e sua viabilização via financiamento coletivo. A articulação de diferentes stakeholders se mostrou como alternativa válida e, talvez, como fator essencial para a inovação.

\section{A INOVAÇÃO NO CENÁRIO CONTEMPORÂNEO}

O Manual de Oslo, publicação da OECD - Organization for Economic Cooperation and Development - Organização para Cooperação e Desenvolvimento Econômico (em tradução livre) descreve inovação como "a 
implementação de um produto (bem ou serviço) novo ou significativamente melhorado, ou um processo, ou um novo método de marketing, ou um novo método organizacional nas práticas de negócios, na organização do local de trabalho ou nas relações externas" (OECD, 2005, p. 55).

A publicação estabelece quatro tipos de inovações que abrangem a realidade empresarial: inovações de produto, inovações de processo, inovações organizacionais e inovações de marketing. A inovação de produto se baseia na inserção de um bem ou serviço novo - ou significativamente aprimorado - no mercado partindo da utilização de novos conhecimentos ou tecnologias ou da combinação de conhecimentos e tecnologias já existentes. A inovação de processo consiste na implementação de novos métodos de produção ou distribuição, ou métodos significativamente melhorados que possam reduzir custos de produção ou distribuição, melhorar a qualidade do processo e até produzir ou distribuir novos produtos ou produtos aprimorados. A inovação de marketing implementa um novo método de marketing que altera a concepção do produto, sua embalagem, posicionamento, promoção ou fixação de preço. As mudanças devem fazer parte de um novo método de marketing que ainda não tenha sido utilizado pela empresa. A inovação organizacional se baseia na implementação de um novo método organizacional na organização do local de trabalho, nos negócios ou nas relações externas, visando a melhoria do desempenho da empresa. Assim como a inovação em marketing, a mudança deve ser resultado de decisões estratégicas tomadas pela gerência e que não tenham sido utilizadas anteriormente na empresa.

Todavia, projetar é algo que o ser humano faz desde a Pré-História (CABEZA e MOURA, 2014). Da Pré-História até a Sociedade Rural, o produtor, seja ele agricultor ou artesão, era o consumidor de sua produção. Na Era industrial, esses papéis se separam pela primeira vez na trajetória do homem social (DE MASI, 2000). A indústria concentrou em suas mãos a decisão do que produzir em escala. As possibilidades de inovação, por sua vez, também ficaram à mercê da indústria (CABEZA e MOURA, 2014).

\footnotetext{
${ }^{1}$ Stakeholder é um termo cunhado por Robert Edward Freeman, filósofo americano e professor de administração. Em seu livro A stakeholder approach to strategic management (1984), o autor afirma que "o stakeholder, em uma organização, é (por definição) qualquer grupo ou indivíduo que pode afetar ou é afetado pela realização dos objectivos da organização" (tradução nossa, p. 40). Os stakeholders podem ser colaboradores, clientes, comunidade, governo, etc. São pessoas ou grupos que legitimam as ações de uma organização.
} 
No entanto, o século XXI é a era das redes colaborativas (MANZINI, 2014). A indústria já não é mais o maior setor da economia e as pessoas já demonstram iniciativa em produzir o que é de interesse próprio ou comunitário. Há uma necessidade urgente de promover novos modelos econômicos, novos sistemas de produção e novas ideias de bem-estar. Definir e implementar estes novos modelos não é uma tarefa simples, porém não é impossível. De fato, durante as duas últimas décadas, a multiplicidade de atores sociais, tais como instituições, empresas, organizações nãolucrativas, cidadãos e suas associações, tem provado a eles mesmos sua capacidade de agir fora dos modelos econômicos tradicionais e, ao fazê-lo, fornecem referências que podem atender às preocupações mais urgentes e aos desafios deste tempo (MANZINI, 2011).

O design, desde sua origem, criou pontes entre a sociedade e a tecnologia. Hoje, o escopo das atividades do designer se expande, vai além de "projetar" e passa a incluir o desenvolvimento de iniciativas de design e a construção uma cultura de design. Novas ideias em bem-estar devem suprir a tensão humana no sentido de aumentar a qualidade de vida. E para isso é preciso envolver as pessoas: visualizar, explorar, prototipar junto delas, trabalhando com sinergia (MANZINI, 2014).

Dois fenômenos relacionados estão presentes no cenário contemporâneo: o crowdsourcing e o crowd-design. Eles são inovações de processo, ou até organizacionais, que podem dar origem a outras inovações de processo, de produto ou de marketing.

\section{O CROWDSOURCING E O CROWD-DESIGN}

Hoje, a conectividade em rede permite que o processo de desenvolvimento de produtos seja aberto à participação dos stakeholders por meio da internet. É desta participação em massa que tem origem o termo "crowd", que pode ser traduzido para a língua portuguesa como "multidão".

O crowdsourcing pode ser considerado um modelo estratégico para atrair um público interessado ou motivado de indivíduos capazes de fornecer soluções superiores em qualidade e quantidade para aqueles que mesmo as formas tradicionais negócio podem fazer (BRABHAM, 2008). É uma ação de terceirização de forma colaborativa e aberta, como convite, um trabalho que originalmente seria feito por um profissional e, em geral, isso 
acontece por meio da internet (DICKIE et al, 2014). O crowdsourcing, de certa forma, se assemelha à abertura do processo de inovação de uma empresa, por integrar diversas competências individuais (pessoas criativas, cientistas, engenheiros, etc) ou comunidades organizadas (CHANAL e CARON-FASAN, 2008). Desta forma, é possível notar que a cooperação entre criadores e utilizadores caracteriza o desenvolvimento de novos produtos dentro do conceito de crowdsourcing. Os maiores objetivos do crowdsourcing são: a aprendizagem e a colaboração em rede; a pesquisa e o desenvolvimento; o próprio desenvolvimento e melhoria de produtos e serviços; a pesquisa de opinião entre os stakeholders; a resolução efetiva de problemas e a avaliação de relevância do projeto por meio da demonstração de interesse dos stakeholders (DICKIE e SANTOS, 2014).

Outras ações têm origem a partir do crowdsourcing, entre eles, crowdfunding, crowdvoting, crowdlabor e o crowdstorm (SHOYAMA et al, 2014). $O$ crowdfunding é um financiamento coletivo que busca doadores ou investidores informaise individuaisque possam aplicar determinadas quantias de dinheiro para a viabilização de um projeto específico (BANNERMAN, 2013). Ele vai na contramão do modelo tradicional de investimento privado por recrutar o financiamento público de um projeto e não o financiamento por parte do grande investidor ou de um pequeno grupo de investidores (BELLEFLAMME et al, 2011 apud DICKIE et al, 2014). O crowdvoting busca a opinião para determinada etapa de decisão num projeto de criação colaborativa (DJELASSI e DECOOPMAN, 2013). O crowdlabor, por sua vez, consiste na distribuição de tarefas de um projeto entre aqueles dispostos a colaborar com a realização destas, desde as mais simples às mais complexas de se realizar (CROWDSOURCING.ORG, 2010). Por fim, o crowdstorm, é um processo de geração de ideias realizado na comunidade interessada que pode ter graus de complexidade distintos, coletando ideias, selecionandoas e até aprimorando-as (ABRAHAMSON, 2013).

A colaboração em massa permite que as pessoas se tornem pequenos produtores de soluções e ingressem no mercado até então dominado pelas grandes empresas. O envolvimento comunitário no processo de desenvolvimento de produtos é o chamado "crowd-design". O crowddesign é uma modalidade recente de projeto e de produção que usa os conhecimentos e recursos disponíveis na comunidade para projetar soluções. É por meio das interações entre indivíduo, comunidade e 
instituições que será mais fácil viabilizar soluções inovadoras e sustentáveis para problemas de aprendizagem, bem-estar, saúde, mudanças de mentalidade social e política, entre outros problemas. Ele pode reduzir o tempo do desenvolvimento de projetos, diminuir os custos e criar redes de suporte à implementação e manutenção das soluções desenvolvidas (DICKIE et al, 2014). A utilização do crowdsourcing no desenvolvimento de novos produtos dentro das organizações integra o consumidor (que pode ser considerado um desenvolvedor externo) no processo de inovação, fazendo com que o consumidor se torne parte do processo. Esta oportunidade eleva a autoestima do indivíduo, que está sendo ouvido durante o processo e garante à organização o projeto de produtos melhor adaptados às necessidades deste consumidor (DJELASSI e DECOOPMAN, 2013 apud OLIVEIRA, DICKIE e SANTOS, 2015).

Para demonstrar a importância da interação e colaboração entre stakeholders em um projeto, será apresentado um case de design premiado, o Eatwell.

\section{CASE - EATWELL}

\subsection{O PRODUTO}

Eatwell é um conjunto de mesa para pessoas com deficiências cognitivas, como doença de Alzheimer e demência (Figura 1). O projeto ganhou o 10 lugar no Design Challenge "Maximizing Independence for those with cognitive impairment" (Maximização da independência para aqueles com comprometimento cognitivo, em tradução livre) promovido pelo Stanford Center on Longevity.

Figura 1: O conjunto de mesa Eatwell

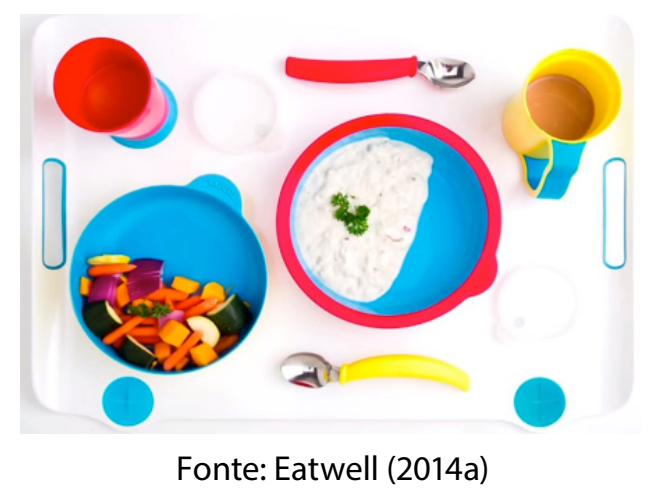


Além das dificuldades motoras, pessoas com deficiências cognitivas também têm dificuldades de distinguir alimentos e bebidas nos recipientes. De acordo com Eatwell (2014ab), a Universidade de Boston constatou que cores brilhantes aumentam o consumo de alimentos em $24 \%$ e o de líquidos em $84 \%$. Partindo deste resultado, o projeto escolheu a cor azul para os pratos, pelo contraste com os alimentos, e as cores amarelo e vermelho para estimular o apetite.

Os pratos possuem ângulos de $90^{\circ}$ e têm fundo inclinado para ajudar os usuários a coletar a comida com a colher de forma mais fácil e também impedir que o alimento colhido caia acidentalmente fora do prato. As colheres foram projetadas para coincidir com a curvatura dos pratos (Figura 2).

Figura 2: Pratos e colheres do Eatwell

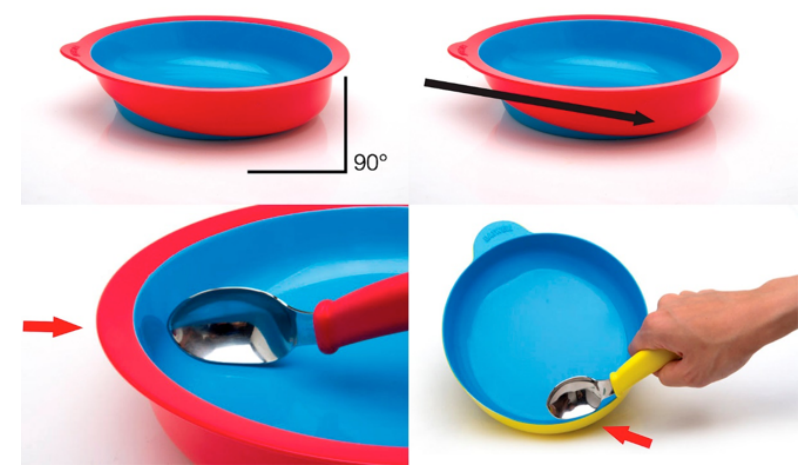

Fonte: Eatwell (2014a)

O copo possui uma base de borracha que atua como estabilizador. A caneca possui uma alça que se estende até sua base como suporte adicional. A alça é também especialmente projetada para auxiliar os usuários com artrite (Figura 3).

Figura 3: Copo e caneca do Eatwell

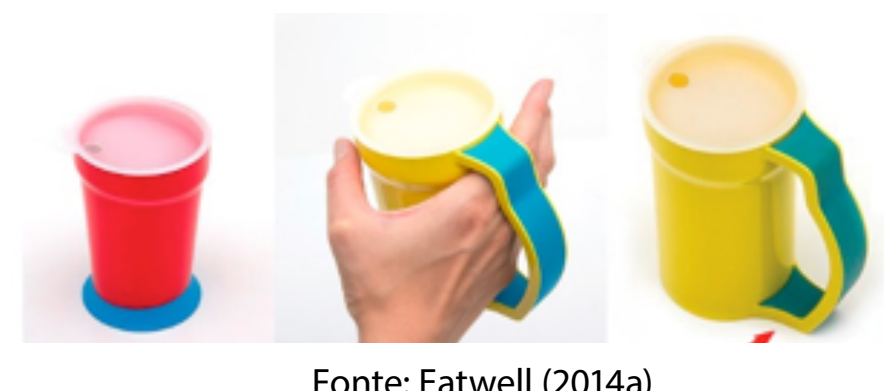

Fonte: Eatwell (2014a) 
Todos os produtos possuem material anti-deslizante na parte inferior (Figura 4).

Figura 4: Vista lateral do conjunto de mesa Eatwell

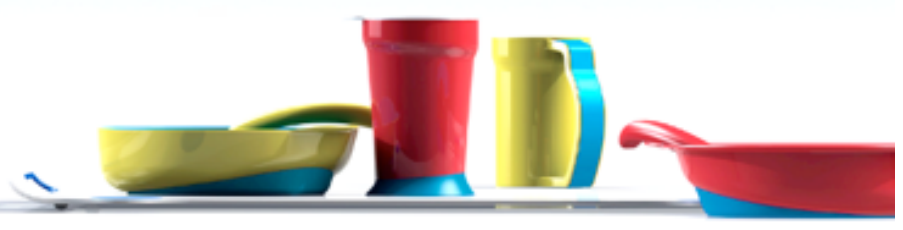

Fonte: Eatwell (2014a)

O conjunto também vem com uma bandeja que permite aos usuários usar um babador para ajudar a recolher qualquer alimento que caia fora do prato (Figura 5).

Figura 5: Vista superior da bandeja

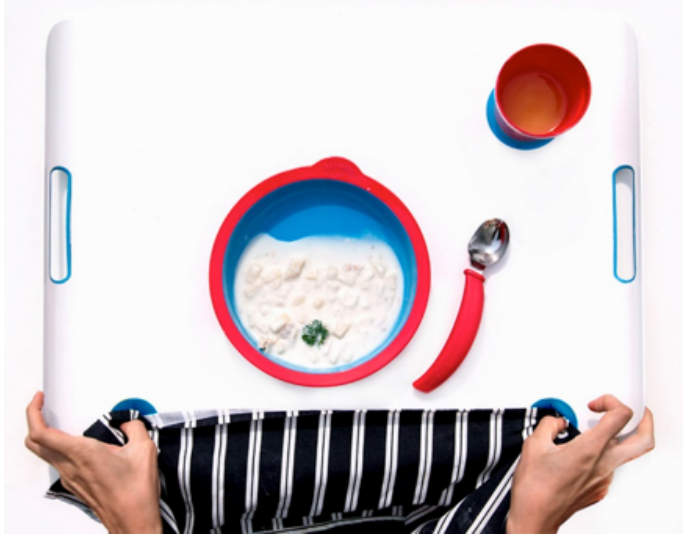

Fonte: Eatwell (2014a)

\subsection{OS STAKEHOLDERS DO PROJETO}

Para compreender a origem e a viabilização produtiva do projeto, seus stakeholders serão apresentados.

A desenvolvedora do projeto é Sha Yao, designer de São Francisco, Califórnia. Durante sua adolescência ela ajudou a cuidar de sua avó, que teve a doença de Alzhiemer, e isso a motivou a pesquisar maneiras de facilitar a vida dos idosos doentes e dos seus cuidadores. Enquanto estava cursando a graduação, ela foi voluntária num day-care center para idosos para observar a rotina, entrar em contato direto com as necessidades deles e poder projetar algo relevante para o dia a dia. A convivência e troca de experiências entre a designer, os cuidadores e os idosos direcionou o projeto para objetos que pudessem tornar 
as refeições mais fáceis e dignas para estes idosos. Os usuários diretos do produto são os idosos com deficiências cognitivas, enquanto os cuidadores são aqueles que compreendem de perto às necessidades dos idosos e que, podem muitas vezes, saber expressá-las melhor em palavras, além de terem a experiência na rotina de auxiliar os idosos em sua alimentação, o que explica a importância destes stakeholders no direcionamento e desenvolvimento do projeto. Sha Yao, liderou o desenvolvimento num formato de crowd-design, com a colaboração dos cuidadores e dos idosos, que podem ser considerados desenvolvedores externos deste projeto. Enquanto o papel do público alvo do projeto foi auxiliar na definição do produto, relatando necessidades, testando protótipos (Figura 6), o papel da designer foi materializar soluções para esta necessidade, de acordo com parâmetros de produção, visando a viabilidade produtiva deste projeto.

Figura 6: Protótipos de teste
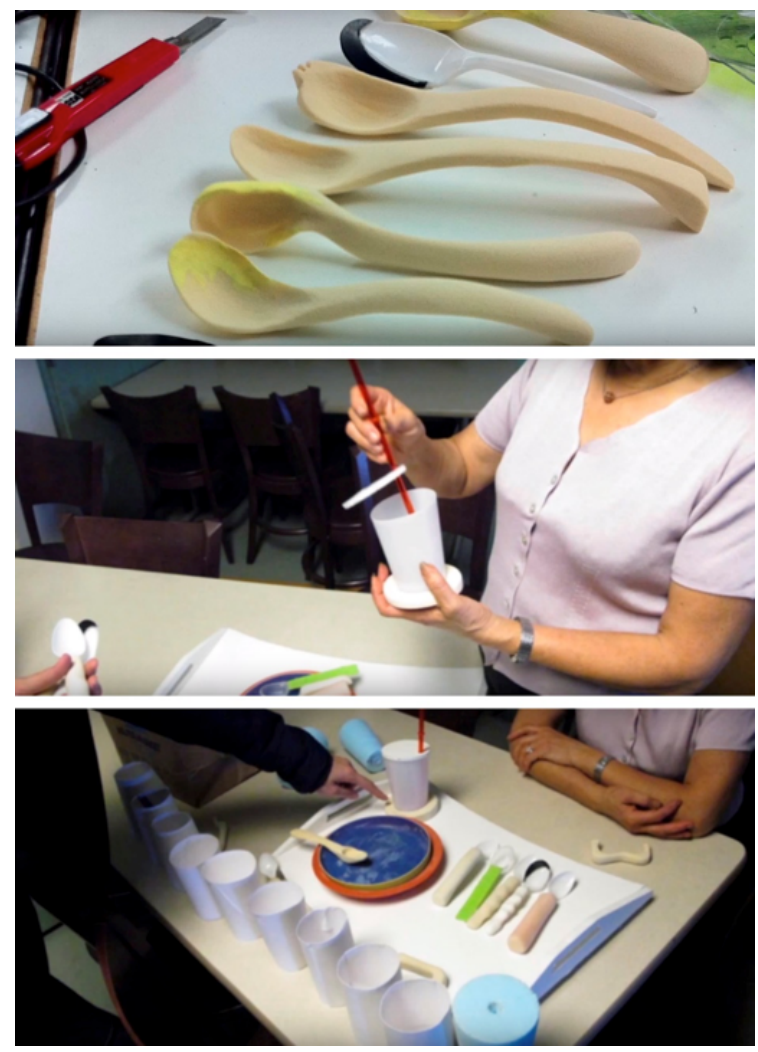

Fonte: Eatwell (2014b)

A viabilização produtiva do projeto pôde ser encaminhada, quando o Stanford Center on Longevity lançou o desafio de design "Maximizing 
Independence for those with cognitive impairment" em parceria com a Aging 2.0. O Stanford Center on Longevity pesquisa formas inovadoras para a solução de problemas daqueles com mais de 50 anos. O intuito é, por meio da ciência e da tecnologia, melhorar o bem-estar destas pessoas. A Aging 2.0 é uma plataforma global de inovação voltada para atender às demandas do idoso. Sua missão é acelerar os projetos inovadores voltados à melhoria de vida dos idosos.

Dentre 52 equipes de 15 países, o Eatwell conquistou o $1^{\circ}$ lugar no desafio e passou a receber suporte do Stanford Center on Longevity e da Aging 2.0 (Figura 7).

Figura 7: Idoso utilizando o conjunto de mesa Eatwell
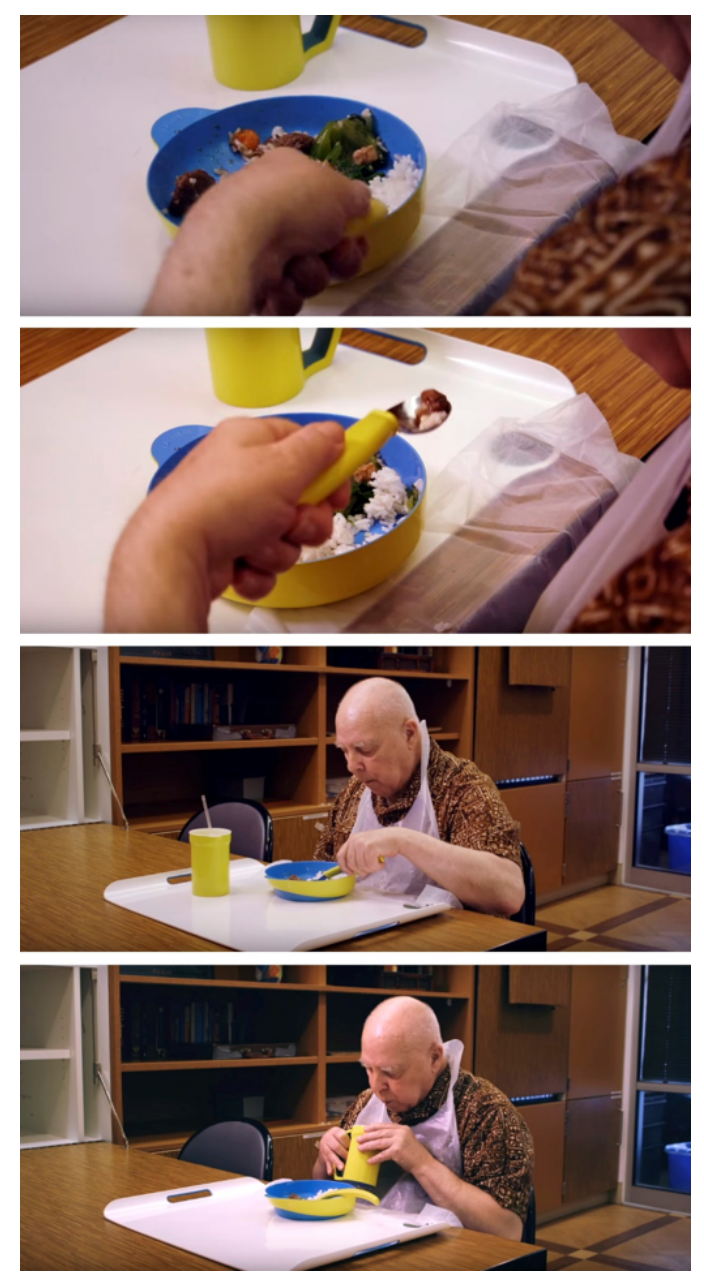

Fonte: Fonte: Eatwell (2014b)

O projeto, então, seguiu para uma plataforma de crowdfunding, a Indiegogo. 
A campanha foi lançada em 21/10/14 com o objetivo de arrecadar U\$70.000. E foi finalizada em 20/12/14 com U\$76.063 - 109\% da meta.

Dada a viabilidade financeira do produto, o projeto seguiu em busca de certificações para comercialização dentro dos Estados Unidos e no exterior, incluindo os testes laboratoriais dos materiais utilizados para garantir a segurança no uso.

Os materiais utilizados são aço inoxidável para as colheres, PP (polipropileno - polímero termoplástico reciclável), TPE (elastômero termoplástico composto também conhecido como borracha termoplástica) e silicones aprovados pela FDA (Food and Drug Administration - órgão governamental dos EUA responsável pelo controle de alimentos, suplementos alimentares, medicamentos, cosméticos, equipamentos médicos, entre outros). Os produtos são livres de substâncias nocivas à saúde como BPA (Bisfenol-A composto usado na produção de plásticos e resinas) e ftalatos (são os ésteres de ácido ftálico utilizados como plastificantes, substâncias adicionadas ao plástico para aumentar sua flexibilidade, transparência, durabilidade, longevidade) e seguem a RoHS (Restriction of Certain Hazardous Substances ou Restrição de Certas Substâncias Perigosas em tradução livre - um conjunto de normas europeu que proíbe o uso de certas substâncias perigosas em processos de fabricação de produtos: cádmio $(\mathrm{Cd})$, mercúrio $(\mathrm{Hg})$, cromo hexavalente $[\mathrm{Cr}(\mathrm{VI})]$, bifenilos polibromados (PBBs), éteres difenilpolibromados (PBDEs) e chumbo (Pb)). A figura a seguir (Figura 8) apresenta de forma sintética as relações de colaboração entre os stakeholders.

Figura 8: Relações de colaboração entre os stakeholders do projeto

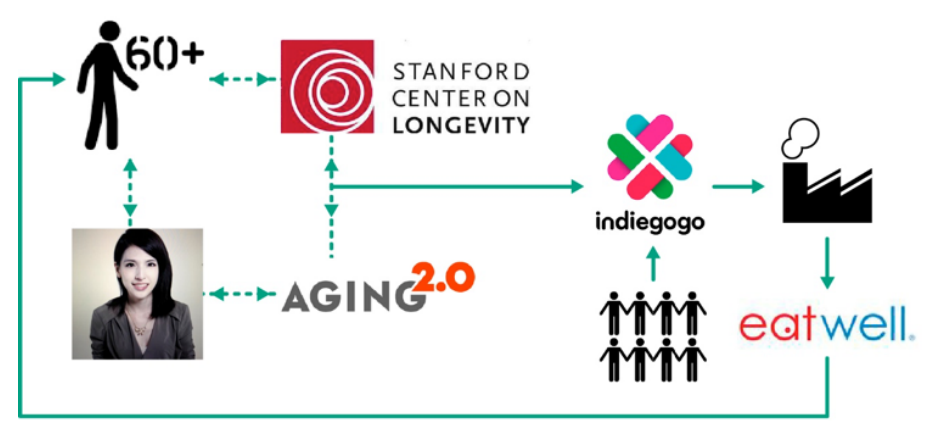

Fonte: própria

A idealização e boa parte do desenvolvimento do Eatwell nascem da colaboração entre a designer, Sha Yao, os cuidadores, os idosos, o Stanford 
Center on Longevity e a Aging 2.0. O financiamento do projeto via crowdfunding permitiu a produção em série do produto e a consequente distribuição e atendimento aos idosos e aqueles com deficiências cognitivas. A indústria fez parte desta cadeia, mas não na posição de líder de inovação, como geralmente acontece. Este papel ficou a cargo dos atores, que tinham empatia pela causa e souberam prospectar cenários - a designer, os cuidadores, os idosos, o Stanford Center on Longevity e a Aging 2.0.

\section{CONSIDERAÇÕES FINAIS}

A contemporaneidade coloca o ser humano no centro do projeto e faz com que este tenha que ser compreendido como sujeito e ser social. A complexidade do cenário atual, devido ao crescente volume de informações, aos problemas cada vez mais transversais e multidimensionais e às interações entre o tangível e o intangível, faz com que o exercício do design e da inovação necessitem da articulação entre atores para a compreensão do contexto. O pensamento projetual precisa ser mais amplo e consistente para atender aos pré-requisitos requisitos de sustentabilidade ambiental, social e econômica, respeitando identidades culturais e minorias. Cabe ao designer, então, o raciocínio analítico e reflexivo para projetar junto ao seu público no século XXI.

O Eatwell é um exemplo de design centrado no usuário e um produto inovador, pois facilita a ingestão de alimentos por parte dos idosos e, ao mesmo tempo, reduz a carga da tarefa da alimentação por parte dos cuidadores, tornando o processo de comer mais digno. Não há nenhuma tecnologia ou material inovadores aplicados neste projeto, entretanto seu potencial inovador não passa despercebido: sua concepção, seu desenho e seu processo de viabilização via financiamento coletivo. A articulação de diferentes stakeholders se mostra como alternativa viável, interessante e, talvez, necessária para a inovação no cenário contemporâneo.

\section{BIBLIOGRAFIA}

ABRAHAMSON, Shaun. Crowdstorming: three patterns for crowdsourcing brainstorming. 2013. Disponível em: <http://www.crowdsourcing.org/editorial/ crowdstorming-three-patterns-for-crowdsourcingbrainstorming/23490>. Acesso em: 17 abr. 2016.

BANNERMAN, S. Crowdfunding Culture. In: Journal of Mobile Media, v. 7, n. 1, p. 1-30, 2013. 
BRABHAM, Daren C. Crowdsourcing as a model for problem solving: an introduction and cases. Convergence: The International Journal of Research into New Media Technologies, v. 14, n. 1, p. 75-90, 2008.

CABEZA, Edison U. R.; MOURA, Mônica. OPEN DESIGN: abertura + design = prática projetual para a transformação social. In: Congresso Brasileiro de Pesquisa e Desenvolvimento em Design, 11., 2014, Gramado. Anais... Gramado: Universidade Federal do Rio Grande do Sul, 2014, p. 2719-2730.

CHANAL, Valérie; CARON-FASAN, Marie-Laurence. How to invent a new business model based on crowdsourcing: the Crowdspirit case. In: Conférence de I'Association Internationale de Management Strat'egique, 2008, Sophia-Antipolis, France. Anais... Sophia-Antipolis, France, 2008, pp.1-27, 2008.

CROWDSOURCING.ORG. Cloud labor. 2010. Disponível em: <http://www. crowdsourcing.org/community/cloud-labor/6>. Acesso em: 17 abr. 2016.

DE MASI, Domenico. O Ócio Criativo. Tradução por Léa Manzi. Rio de Janeiro: Sextante, 2000.

DICKIE, Isadora B. et al. O Processo de Desenvolvimento De Produtos Via CrowdDesign: oportunidades para a sustentabilidade. In: Congresso Brasileiro de Pesquisa e Desenvolvimento em Design, 11., 2014, Gramado. Anais... Gramado: Universidade Federal do Rio Grande do Sul, 2014, p. 1268-1279.

DICKIE, Isadora B.; SANTOS; Aguinaldo dos. Bases para Entendimento do Crowdsourcing e sua Aplicação em Projetos de Crowd-design. In: Congresso Brasileiro de Pesquisa e Desenvolvimento em Design, 11., 2014, Gramado. Anais... Gramado: Universidade Federal do Rio Grande do Sul, 2014, p. 1256-1267.

DJELASSI, Souad; DECOOPMAN, Isabelle. Customers' participation in product development through crowdsourcing: Issues and implications. In: Industrial Marketing Management, v. 42, n. 5, p. 683-692, 2013.

EATWELL. Eatwell: tableware for people with Special need/Alzhiemer's. 2014a. Disponível em: <http://www.eatwellset.com>. Acesso em 15 out. 2015.

Eatwell Tableware Set. 2014b. Disponível em: <https://www.youtube. com/watch? $v=P h I Q R I 2 D 9 c 0>$. Acesso em 15 out. 2015.

FREEMAN, Robert E. Strategic Management: a stakeholder approach. Boston: Pitman Series in Business and Public Policy, 1984.

MANZINI, Ezio. Design in a changing world: in the age of networks and sustainability. Palestra proferida no $11^{\circ}$ Congresso Brasileiro de Pesquisa e Desenvolvimento em Design em 30 set. 2014.

. The New Way of the Future: Small, Local, Open and Connected. 2011. Disponível em: <https://centres.smu.edu.sg/lien/files/2013/10/SocialSpace2011The-New-Way-of-the-Future-Small-local-open-and-connected-Ezio-Manzini-. pdf $>$. Acesso em: 22 out. 2015. 
OECD. Manual de Oslo: diretrizes para coleta e interpretação de dados sobre inovação. 3.ed. 2005. Disponível em: <http://www.mct.gov.br/upd_ blob/0026/26032.pdf>. Acesso em 15 out. 2015.

OLIVEIRA, Alexandre de; DICKIE, Isadora B.; SANTOS, Aguinaldo dos. Crowd-design como alternativa de produção distribuída: um estudo de caso ex-post-facto em empresa de móveis em pallet. In: MIX Sustentável, v. 1, n. 2, p. 48-57, 2015.

SHOYAMA, Thayenne et al. Elaboração do desafio no processo de Crowd Design do projeto Sustainability Maker Brazil. In: GAMPI Plural, 4., 2014, Joinville. Anais... Joinville: Univille, 2014, p. 343-355.

Paula da Cruz Landim Graduada em Arquitetura e Urbanismo pela Faculdade de Arquitetura e Urbanismo da Universidade de São Paulo (1987), mestre em Geografia pelo Instituto de Geociências e Ciências Exatas da Universidade Paulista Júlio de Mesquita Filho, campus Rio Claro/SP (1994), doutora em Arquitetura e Urbanismo pela Faculdade de Arquitetura e Urbanismo da Universidade de São Paulo (2001), estágio de pós-doutorado na Universidade de Arte e Design de Helsinque na Finlândia (2006-2007), Livre-docente em Design de Produto pela Faculdade de Arquitetura, Artes e Comunicação da Universidade Estadual Paulista Júlio de Mesquita Filho, campus de Bauru/SP (2009). Professora do Departamento de Design da Faculdade de Arquitetura, Artes e Comunicação da Universidade Estadual Paulista Júlio de Mesquita Filho, campus de Bauru/SP, desde 1988 e do Programa de Pós-Graduação em Design na mesma universidade desde 2004. Atualmente trabalha nas seguintes linhas de pesquisa: Desenho do Objeto, Projeto de Mobiliário, História do Design e Teoria e Crítica do Design. Possui diversos artigos publicados em periódicos especializados, trabalhos em eventos, tanto nacionais como internacionais, nas áreas de Arquitetura e Design, assim como livros e capítulos de livros publicados.

Recebido em: 29/02/2016; Aceito em: 24/04/2016.

Esta obra foi licenciada com uma Licença Creative Commons.
Possui ainda orientandos de graduação, iniciação científica, mestrado e doutorado na área de Design. Faculdade de Arquitetura, Artes e Comunicação da Universidade Estadual Paulista Júlio de Mesquita Filho, campus de Bauru/SP, desde 1988 e do Programa de Pós-Graduação em Design na mesma universidade desde 2004. Atualmente trabalha na 
S seguintes linhas de pesquisa: Desenho do Objeto, Projeto de Mobiliário, História do Design e Teoria e Crítica do Design. Possui diversos artigos publicados em periódicos especializados, trabalhos em eventos, tanto nacionais como internacionais, nas áreas de Arquitetura e Design, assim como livros e capítulos de livros publicados. Possui ainda orientandos de graduação, iniciação científica, mestrado e doutorado na área de Design.

Lílian Lago Graduada em Design Gráfico pela Universidade Estadual de Londrina, especialista em Gestão Estratégica de Design pela mesma universidade e mestranda do Programa de Pós-Graduação em Design Linha de Pesquisa Planejamento de Produto da Faculdade de Arquitetura, Artes e Comunicação da Universidade Estadual Paulista Júlio de Mesquita Filho. Membro do Grupo de Pesquisa Design Contemporâneo: sistemas, objetos e cultura. Tem experiência em Design, com ênfase em desenvolvimento de produtos, estratégia e design editorial. Foi designer da Editora da Universidade Estadual de Londrina e analista de negócios em design, tecnologia e inovação no SENAI Paraná. Possui publicações como os capítulos de livro "Ensino e Pesquisa Científica no Design e na Moda no Brasil: caminhos que se cruzam e se realimentam" (In: Maria de Fátima da Silva da Costa Mattos. (Org.). Pesquisa e formação em Moda. 1ed. São Paulo: Estação das Letras e Cores, 2015) e "Design Contemporâneo: pesquisas interdisciplinares" (In: Luis Carlos Paschoarelli e Rosio Fernández Baca Salcedo. (Org.). Interação: panorama das pesquisas em Design, Arquitetura e Urbanismo. 1ed. Bauru: Canal 6, 2015), artigos publicados em periódicos como "A Pesquisa em Design e a Interdisciplinaridade" (In: Revista Educação Gráfica, v. 19, p. 106-123, 2015) e artigos aceitos como "Design and fashion processes and methods in the contemporary scene" (In: $3^{\circ} \mathrm{CIMODE}$ - Congresso Internacional de Moda e Design, 2016, Buenos Aires, Argentina). Atualmente é bolsista da CAPES e pesquisa processos e métodos de design na contemporaneidade.

Juliana Cristina de Angelo Graduada em Educação Física pela Faculdade de Ciências da Universidade Paulista Júlio de Mesquita Filho, campus 
Bauru/SP, e mestranda do Programa de Pós-Graduação em Design Linha de Pesquisa Ergonomia pela Faculdade de Arquitetura, Artes e Comunicação da Universidade Estadual Paulista Júlio de Mesquita Filho. Membro do grupo de pesquisa do Laboratório de Informação, Visão e Ação (LIVIA), liderado pelo Prof. Dr. Sérgio Tosi Rodrigues. Tem experiência em Educação Física. Como educador físico trabalha com reabilitação física e condicionamento e nos seguintes temas: movimento dos olhos, controle postural e condução simulada de veículos. Possui publicações em congresso e apresentações como "Influences of open and closed on posture balance body during the range of na object'" e "A percepção do usuário no âmbito da Ergonomia: um estudo bibliométrico" (In: IDEMI 2015 - IV Internacional Conference on Integration of Design, Engeneering and Management for Innovation, 2015, Florianópolis/SC). Apresentações em congresso como: "Comportamento dos olhos e da postura em adultos jovens" (In: VII Congresso Brasileiro de Comportamento Motor, Belo Horizonte/MG, 2014), "Movimento dos olhos em diferentes bases de apoio em adultos jovens e crianças" (In: 4 Encontro Paulista de Comportamento Motor, Ribeirão Preto/SP, 2015), "Influências das posturas abertas e fechadas sobre o controle postural" na modalidade de painel científico (V Simpósio do Programa de Pós-Graduação em Psicologia do Desenvolvimento e Aprendizagem, realizado na FC da Unesp/Bauru em 2015. Atualmente desenvolve a pesquisa "Atenção Visual e condução simulada de veículos sobre o efeito da aptidão física e da velocidade".

Fabrício Mira Graduado em Desenho Industrial com Habilitação em Programação Visual pela Faculdade de Arquitetura, Artes e Comunicação da Universidade Estadual Paulista Júlio de Mesquita Filho, campus de Bauru/SPem 2006, e mestrando do Programa de Pós-Graduação em Design - Linha de Pesquisa Planejamento de Produto pela mesma universidade. Membro do grupo de Pesquisa em Design Contemporâneo: sistemas, objetos e cultura. Tem experiência nas áreas de Design e Artes, com ênfase nos seguintes temas: Design Gráfico, Identidade Visual, Ergonomia, Artes Plásticas, Ilustração Digital 2D/3D, llustração Não-Digital, Realidade Virtual e Histórias em Quadrinhos. Atuou como professor de ensino superior na 
Graduação do curso de Artes e na Pós-Graduação do curso de Letras e Artes - Interlinguagens do Texto e da Imagem, pelas Faculdades de Dracena/SP, mantidas pela Fundação Dracenense de Educação e Cultura - FUNDEC. Possui publicações em capítulo de livro e anais de congresso como "A Ergonomia aplicada ao design de jornais - um estudo de caso dos jornais de Bauru- SP" (In: Estudos em Design nas Universidades Estaduais Unesp e Usp. 1 ed. São Paulo: Editora UNESP, 2006. v. 1. 308p.) e "Contribuições do Design para o uso de realidade virtual no tratamento da síndrome do pânico" (In: IDEMI 2015 - IV Internacional Conference on Integration of Design, Engeneering and Management for Innovation, 2015, Florianópolis/SC). Atualmente é bolsista da CAPES e desenvolve um ambiente virtual para o tratamento da Síndrome do Pânico por meio de realidade virtual para o Laboratório do Pânico e Respiração da UFRJ. 